\title{
SPECIFIC 2-D SPECTRAL ESTIMATION FOR WIDEBAND BEAMFORMING
}

\author{
M.A. Lagunas, G. Vázquez, J.F. Sitjar
}

E.T.S.I. Telecomunicación, T.S.C. Dept. Apdo. 30.002, 08080 Barcelona, Spain

\section{ABSTRACT}

This paper presents the potential of a 2-D spectral estimation approach to the problem of DOA estimation in wideband array beamforming problems. It is shown that the covariance or interspectral matrix does not provides the adequate starting point in order to coherently average the energy of a given source. Angle focussing is envisaged from a prior two-dimensional spectral density. From this prior estimate basic DFT interpolation may be used to obtain a new angle/frequency representation of the wavefield, where source focussing can be done without requiring ad-hoc transformations. The new viewpoint of the wideband DOA estimation problem states more clearly the potential, objectives and the fundamental limitations of focussing methods; regardless, they are carried out at the interspectral matrix level or at the 2-D spectral density level.

\section{INTRODUCTION}

DOA estimation in wideband arrays is a problem of finding patterns in a 2-D spectral estimate formed by the representation of density of energy with respect (time) frequency $\omega$ and spatial frequency $\Omega$. Regardless this nature of the problem, many currently reported methods in the field use to handle a reduced model, as the same time reduced view, of the wavefield. This model, named as covariance model or interspectral matrix, is ji'st a reduced information function of the two dimensional periodogram. Nowadays, it is well recognized, in one-dimensional spectral estimation that the acf representation is a very reduced version of the information given by the corresponding periodogram. In other words, in a snapshot of sensors with $\mathrm{N}$ samples per sensor, the correct starting point will be the corresponding periodogram and not a reduced representation given in a $Q \times Q$ matrix.

In this paper, we report a procedure to obtain angle focussed or angle oriented periodograms just by DFT interpolation of the frequency-spatial frequency periodogram. Once the angle focussed is done, the

This work has been granted by the ClCYT National Research Plan on Information and Communication Technologies of Spain. average can be performed in order to obtain an averaged periodogram which represents the 1-D equivalent problem of the wideband source DOA estimation. As it may be viewed no prior information about the sources number, or associated DOAs, is required and, as a consequence of DFT interpolation, the procedure shares the advantages of unitary transformations for matrix focussing with lower computational load. Finally, it is shown that the reported framework allows to obtain very in deep the involved problems of coherently averaging enery in the wideband DOA problem.

\section{THE COVARIANCE MODEL AND FREQUENCY FOCUSSING}

The covariance model for a wavefield of $s$ sources, sensed by an aperture of $Q$ elements, temporally sampled with $L$ samples per element, is given in terms of the Fourier transform of each signal, lets say with $\mathrm{N}$ (greater than $L$ ) samples or frequency bins. The two dimensional snapshot is arranged in a single vector $x(w)$ by seting the frequency content in the successive components of $x(\omega)$ for all the sensors elements contained in the aperture.

$$
\underline{x}(w)=\underline{A}(w) \underline{x}_{S}(w)-\underline{n}(w)
$$

Being $A(w)$ the matrix containing the DOAs of the impinging sources, $\underline{X}_{S}(w)$ the vector containing the Fourier transform of the sources and $\underline{n}(w)$ the noise frequency vector. The covariance model, also refered as interspectral array matrix $R(w)$, is obtained by averaging, over successive snapshots the product $\underline{x}(w) \underline{x}^{H}(w)$.

$$
\begin{aligned}
\underline{R}(w) & =E\left[X(w) \underline{x}^{H}(w)\right]=\underline{A}(w) \underline{\underline{P}}_{s}(w) \stackrel{A}{A}^{H}(w)+ \\
& +\sigma_{n}^{2} \underline{P}_{n}(w)
\end{aligned}
$$

We will made some remarks about this signal model, the processing involved in its generation, and the associated procedures to obtain the DOAs information from it.

First at all, the model stablishes, at a very first steep, the number of sources; this fact is responsible for many fundamental limitations of the procedures derived from this signal model.

Secondly, this signal model goes only at the midle of the actual size of the problem which is a two dimensional spectral estimation problem. In fact, it is well recognized [1] that periodogram information is much better than reduced acf forms, as the initial or prior information, for spectral estimation, frequency detection or direction of 
arrival estimation. In this sense, it is better to consider the two-dimensional periodogram which is obtained from (1) as denoted in (3)

$$
P(w, w)=\Phi^{H} \cdot X(w) \underline{x}^{H}(w) \phi
$$

where $\phi$ is the vector which explores the DOAs information.

Other point, very important, relates with the average needed to obtain (2). The average is done, at any level in signal processing, just to enhance the signal energy with respect interference and noise. But, concerning DOA determination, it is possible to obtain such enhancement just from a single snapshot, by averaging in the 2-D periodogram along some successive frequency beams.

$$
P\left(w_{0}, \Omega\right)=\sum_{l=-R}^{+R} P\left(w_{1}, \Omega\right)
$$

This approach attemps to enhance the energy from every source at a given spatial frequency $\Omega$. A frequency detector such as MUSIC applied to the data autocorrelation associated to $\mathrm{P}\left(\mathrm{w}_{0}, \Omega\right)$ completes the procedure. The drawback of this technique is the same that promoted the socalled focussing methods [2], [3]. To realice why (5) is refered as an uncoherent averaging procedure note that the angular frequency components in the received snapshot for sensor labeled with index $q$ is :

$$
\Omega(q)=w \frac{d_{q}}{c} \cdot \sin \theta \cdot \cos \left(\varphi-\varphi_{q}\right)
$$

being $\left(d_{q}, \varphi_{q}\right)$ the polar coordinates of sensor labeled with index $q$ in a planar array, $(\theta, \varphi)$ are the angles of arrival, and, $c$ is the sound velocity. The quotient $(\sin \theta) / c$ is refered as the slowness factor. Lets us assume hereafter a linear array (i.e. $\Omega(q)$ independent of $\varphi$ ). These assumptions will not restrict the conclussions of the presentation.

$$
\Omega(q)=w \frac{d_{q}}{c} \sin \theta
$$

From (6) it is clear, that averaging over $\Omega$ does not precludes to average energy bands along an angle of arrival $\theta$, due to the presence of $w$ in this last relationship. In fact, the average done in (4) forces to compute energy from different angles in order to keep constant the angular frequency $\Omega$.

The so-called focussing methods consist on the use of matrix transforms $T$ which applied over successive frequency bins of the interspectral matrix $\underline{\underline{R}}\left(w_{1}\right)$ try to avoid the unalignement implicit in (4). The problem, with such a transformation comes from the signal model involved, and that they do not provide very much insight in what it really is the problem we are facing.
In the next section, we will provide our approach to the wideband DOA estimation and what we may expect from a focussing method. Previously, and in order to better aprice the hereafter reported approach, let us sumarize the inherent difficulties of the currently reported methods, refered as focussing methods (i.e. methods which use matrix transformation in the interspectral signal model).

- Even non-unitary transforms require a previous knowledge of prior estimates of the angles were energy is impinging the array [2].

Unitary transforms require large computational load to evaluate SVD of the interspectral matrix at every selected frequency bin for focussing [3].

- Non-unitary transforms require a generalized SVD of the pencil ( $\underline{\underline{R}}, \underline{R}_{n}$ ) which further increases the computational burden of the procedure. $(\underline{R}(w)$ as defined before and $\underline{R}_{n}$ as $\underset{=}{=}=\frac{T}{=}$ ) [3].

There is not an easy explanation of objectives and transform design due to the signal model used.

Next section will try to overcome these difficulties by reporting an approach based in facing the DOA wideband beamforming as a $2-\mathrm{D}$ spectral estimation problem.

A different approach, refered by array interpolation is reported by Bienvenu et al. in reference [4]. The idea is just to keep independent of the frequency the spatial frequency, by using aperture interpolation for an equally spaced array, at the sensors level. The procedure is performed over the covariance model. This fact, somehow promotes similar difficulties that other procedures, in the sense, that there is not a clear explanation of objectives, in the sense that there is not a clear explanation of objectives, fundamental limitations of the procedure and not easy extension to other array geometries.

\section{ANGLE FOCUSSING IN THE 2-D FREQUENCY PLANE}

We will explore in this section the opportunities provided by the 2-D spectral representation provided by (3), to stablish the objective of focussing methods and to report an easy to compute procedure to reach the mentioned objetive.

Let us assume that we can obtain the optimal spectral density $S(\omega, \Omega)$ instead of a periodogram or Capon estimate. Also, without loss of generality the maximum frequency $f_{m}$ verifies the Nyquist criterium

$$
f_{m}=1 / 2 T
$$

It the sound velocity is independent of frequency, the spatial frequency of the received snapshot depends on the time frequency $\omega$. It is clear from (4) and (6) that the dependency of spatial frequency $\Omega$ in the time frequency $\omega$ forces specific patterns in the actual power spectral density of the wavefiled $S(\omega, \Omega)$. This dependency will be, as a consequence of (6.a) line-patterns in the case of linear 
equally spaced array.

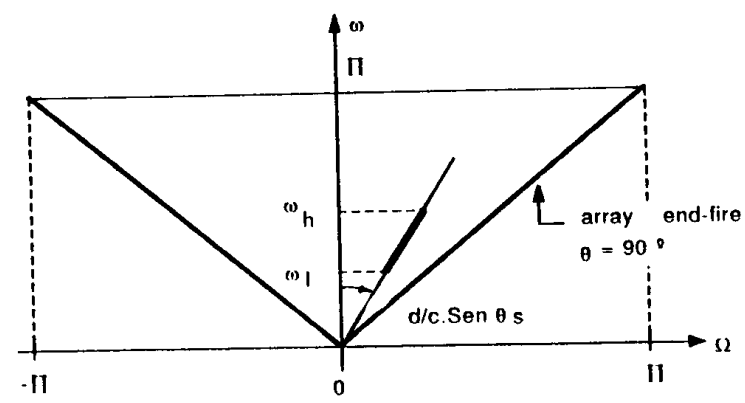

Figure1. 2-D pattern of a broad-band source impinging the equally spaced array from angle $\theta_{S}, d$ is the interspacing elements distance.

Other patterns will show up depending on the geommetry of the array and the dependence of sound propagation with frequency.

The pattern shown in figure 1 is just an ideal situation. More realistic is to describe these patterns in terms of spread of the 2-D periodogram or Capon's method. Figure 2 shows for three sources at $-60^{\circ}, 0^{9}$ and $45^{\circ}$ for 16 sensors with 32 samples per sensor and $(8 \mathrm{KHz}$, $1.43 \mathrm{KHz}),(6.5 \mathrm{KHz}, 1.43 \mathrm{KHz})$ and $(5 \mathrm{KHz}, 1 \mathrm{KHz})$ of central frequency and bandwidth respectively.

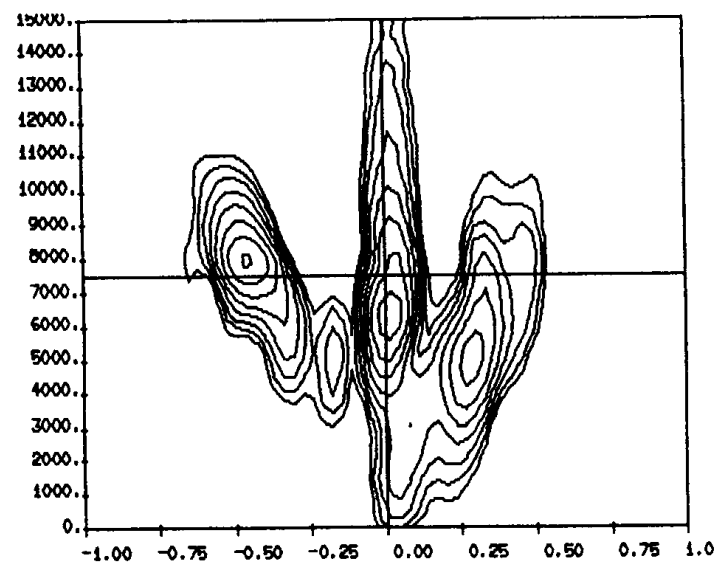

Figure 2. 2-D patterns for three broadband sources in a linear array of 16 sensors, 32 samples/sensor and DOAs equal to $-60,0$ and 45 degrees. Note the linear spread pointing to the origin of the representation.

Other patterns will show up depending on the array geometry and in accordance with the dependence of sound propagation with the time frequency $\omega$.

The problem of focussing resides in coherently average the energy coming from the same angle $\theta_{\mathrm{S}}$ around a central frequency selected as the local maxima, in the $w$ axis, from the 2-D periodogram.

This is the same that to average energy from the a priori pattern we expect to show up in the 2-D periodogram or Capon's estimate. Note that, even in the case of a linear array, the constant DOA patterns will be given by (8)

$$
\Omega=\alpha \cdot \omega / c(\omega)
$$

Let us assume that the FFT along the time samples for every snapshot produces (9), where $\theta_{S}$ is the DOA of the source and $S(\omega T)$ is the fourier transform of the source signal

$$
X(l, q)=S(\omega T) \cdot \exp \left(j \frac{d q}{c} \omega \sin \theta_{S}\right)
$$

being $\omega=2 \pi \mathrm{l} / \mathrm{NT}$ and $\mathrm{T}$ the sampling time.

It is worthwhile to remark that our objective is to obtain a 2-D fourier transform described in terms of time frequency and DOA, instead of spatial frequency. Thus a second DFT defined as (11) will explore the possibility of such a representation.

$$
X(I, s)=\sum_{q=0}^{Q-1} X(I, q) \exp \left(-j \Omega d_{q} / c T\right)
$$

Let us concentrate our attention in the term formed by the two complex exponentials of (9) and (10) as it is shown in (11)

$$
j \frac{d_{q}}{c T}\left(\omega T \cdot \sin \theta_{s}-\Omega\right)
$$

because $\omega T$ is equal to $2 \pi l / N$, whenever the discrete exploration of the spatial frequency $\Omega$ is proportional to the frequency term, we assure an angle foccused representation. In other words, if $\Omega$ is sampled as indicated in (12),

$$
\Omega=2 \pi \mathrm{s} / \mathrm{N} \cdot \mathrm{Q}=\frac{2 \pi}{\mathrm{NQ}} \mathrm{I} \cdot \mathrm{m}
$$

the exponential term reduces to (14).

$$
\mathrm{j} \frac{d_{\mathrm{g}}}{\mathrm{cT}} \frac{2 \pi \mathrm{l}}{\mathrm{N}}\left(\operatorname{sen} \theta_{\mathrm{s}}-\frac{\mathrm{m}}{\mathrm{Q}}\right)
$$

Clearly, the new presentation of the 2-D Fourier Transform along index $\mathrm{I}$ and $\mathrm{m}$ is ready now for averaging in a coherent manner.

$X(I, m)=\sum_{q=0}^{Q-1} S(\omega T) \exp \left[j \frac{d_{q}}{c T} \frac{2 \pi I}{N}\left(\operatorname{sen} \theta_{s}-\frac{m}{Q}\right)\right]$ 
Being the new averaged periodogram as (15)

$$
P\left(1_{0}, m\right)=\sum_{O_{0}-M}^{l_{0}+M} x(1, m)
$$

It is important to note that the second DFT for the case of an equally spaced array reduces to (16)

$$
X(I, m)=\left.\sum_{q=0}^{Q-1} X(I, q) \exp \left[-j \frac{d_{g}}{c T} \frac{2 \pi s}{N Q}\right]\right|_{s=1 . m}=
$$

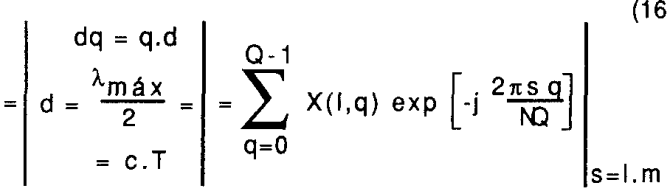

Note that both cases, equally or unequally spaced arrays, require an oversided DFT, which length is determined by the product of the number of DFT bins, in the the time frequency domain, by the number of sensors in the aperture.

The procedure of sampling the oversized spatialfrequency FFT for a linearly equally spaced array is depited in figure 3 . It is important to note how index $m$ is matched to track any source pattern in order to provide energy along the frequency domain for a fixed angle of arrival.

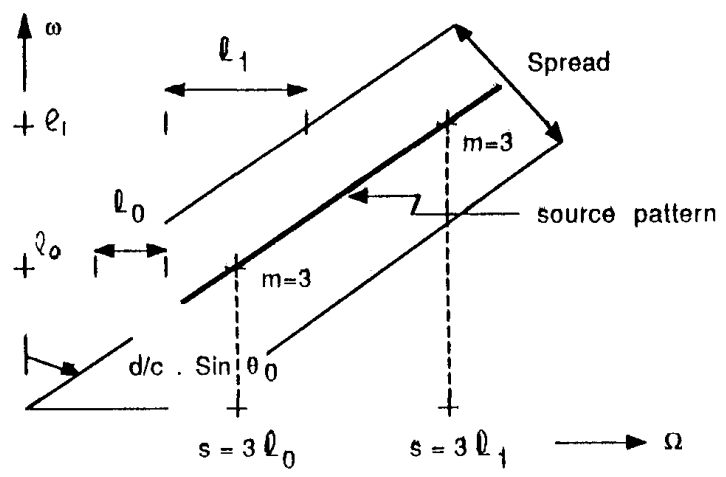

Figure 3. Focussing by sampling oversized DFT in the spatial frequency with sampling rate proportional to the frequency bin.

Note that the procedure, above described, alings the sources along the pattern. Unfortunately the spread along the pattern, as depicted in the figure, does not change with the frequency beam; being responsible or reflecting the fact of the low resolution of small apertures $(\lambda>>d)$.

This is the reason for the focussing loss when using the above procedure or any other one which face the constant spread with frequency or the decrease of resolution when going from high frequency to low frequency beams.

\section{CONCLUSIONS}

The method reported for wideband DOA estimation could be summarized as it is shown below for a linearly equaly spaced array of $\mathrm{N}$ samples per sensor and $\theta$ sensors.

- Perform DFT of $\mathbf{N}$ samples over the time data signal per sensor.

- Perform DFT of NQ samples over the spatial snapshot at every frequency beam $\mathrm{l}=0, \mathrm{~N} / 2$.

- Sample the spatial DFT of NQ samples with rate equal to the index corresponding to the frequency beam.

- Average frequency beams around local maxima of the 2-D focussed periodogram.

- From the resulting averaged periodogram, form the corresponding correlation function and use a frequency detector to obtain the source location in the averaged frequency band.

The procedure just described offerts coherent averaging with potential angles of arrival. Because a DFT interpolation and uniform sampling is used, the noise spectral density in the averaged periodogram remains undistorted. No signal model is assumed and the number of sources is set at a very final steep. Only a basic tool as it is the DFT (or FFT) is used, prior to reduce the DOA estimation, in a wideband problem, to one-dimensional spectral estimation or frequency detection problem.

Future works will describe the above procedure in terms of focussing interspectral matrices and to clarify, up to what degree the spread of line patterns represents a fundamental limitation in the focussing loss. Also, some results on unequally spaced arrays will be reported.

\section{REFERENCES}

[1] El-Jaroudi, J.Makhoul "Discrete pole-cero modeling and applications". Proc. ICASSP-89, vol. 4, pp.21622165, Glasgow, Scotland, May 1989

[2] H. Wang, M. Kaveh, "Coherent signal subspace processing for the detection and estimation of angles of arrival of multiple wide-band sources". IEEE-ASSP, Vol. 33, pp. 823-831, 1985.

[3] H. Wang, M. Kaveh, "On the performance of signal subspace processing - Part II: Coherent wide-band systems". IEEE-ASSP, Vol. 35, pp. 1583-1591, 1987.

[4] G.Bienvenu et al. " Coherent wide band high resolution processing for linear array". Proc. IEEE ICASSP paper U5.2, pp. 2799-2802, Glasgow, Scotland 1989. 